\title{
Effects of Hue, Saturation, and Brightness on Preference
}

\section{Nilgün Camgözz, ${ }^{1 *}$ Cengiz Yener, ${ }^{2}$ Dilek Güvenç ${ }^{3}$}

\author{
${ }^{1}$ Department of Architecture, Faculty of Architecture, Middle East Technical University, 06531 Ankara, Turkey \\ ${ }^{2}$ Department of Interior Architecture and Environmental Design, Faculty of Art, Design, and Architecture, \\ Bilkent University, 06533 Bilkent, Ankara, Turkey \\ ${ }^{3}$ Department of Mathematics, Faculty of Science, Bilkent University, 06533 Bilkent, Ankara, Turkey
}

\begin{abstract}
A study was done to investigate preference responses for foreground-background color relationships. To do this, 123 university undergraduates in Ankara, Turkey, were asked to view eight background colors selected from HSB color space on which color squares of differing hues, saturations, and brightnesses were presented. Subjects were asked to show the color square they preferred on the presented background color. Findings showed that colors having maximum saturation and brightness were most preferred. Blue was the most preferred hue regardless of background. The findings for preferences for foregroundbackground color relationships are also included in this article. (C) 2002 Wiley Periodicals, Inc. Col Res Appl, 27, 199-207, 2002; Published online in Wiley InterScience (www.interscience.wiley. com). DOI 10.1002/col.10051
\end{abstract}

Key words: color preference; color; color combinations

\section{INTRODUCTION}

To prefer is to like better or best. In this study the term preference was considered synonymous with "pleasantness" and "appeal," in the sense the study participants were asked to select the color squares that "suited most to" or "looked good on" the background. Preference, pleasantness, and appeal all suggest subjectiveness. Although preference is to some extent specific to individuals, there are many questions surrounding the complexity of the issue. The extent to which preferences on color combinations reflect personal

\footnotetext{
* Correspondence to: Dr. Nilgün Camgöz, Colour Design Research Centre, School of Engineering, South Bank University, 103 Borough Road, London SE1 0AA, United Kingdom (e-mail: ncamgoz@hotmail.com or camgozn@sbu.ac.uk)

(c) 2001 Wiley Periodicals, Inc.
}

"taste," reflect culture, are universal or biological, and are influenced by fashion trends at the time are all unanswered issues at hand. Despite these variables, many, including Birren, ${ }^{1}$ Chevreul, ${ }^{2}$ Munsell, ${ }^{3}$ and Itten, ${ }^{4}$ have studied color, developing theories by their experiences. In this article these studies are designated as expert views. Note that the expert views did not use controlled-environment setups. Guilford, ${ }^{5}$ Smets, ${ }^{6}$ and Eysenck ${ }^{7}$ did experiments within a controlled environment on color preference, in which color was treated in isolation. Although a single color in isolation may incite preference responses, in actuality colors are rarely viewed in isolation. Color combinations involve more than one color stimulus being perceived simultaneously. This occurrence may evoke visual sensations that differ from those that result from a single color stimulus. Color combinations have not been studied as widely and intensively as colors in isolation. Studies on preferences for color combinations include those by Helson and Lansford ${ }^{8}$ and by Camgöz. ${ }^{9}$ The intention of this study was to explore the effects of hue, saturation, and brightness on preference for colors presented on colored backgrounds.

\section{Research Hypotheses}

The following hypotheses were investigated to reveal foreground-background color relationships:

1. Hue has an effect on preferences for a specific background color.

2. Varying brightness-saturation levels of color samples have an effect on preferences for a specific background color.

3. The hue of a background has an effect on preferences for color samples. 
TABLE I. Brightness-saturation levels that are most preferred on specified backgrounds.

\begin{tabular}{|c|c|}
\hline Background Colo & Preferred Brightness - Saturation \\
\hline Red & BS100 (21\%) \\
\hline Yellow & BS100 (23\%), B75 (17\%), B50 (17\%) \\
\hline Yellow-Green" & BS100 (26\%) \\
\hline Green & BS100 (29\%) \\
\hline Gam & S25 (24\%), BS100 (17\%), S50 (14\%) \\
\hline Blue & BS100 (33\%) \\
\hline Purple & BS100 $(28 \%)$, S $50(26 \%)$ \\
\hline Mugent: & BS100 (22\%) \\
\hline
\end{tabular}

* The mean for BS100 was not singled out in the statistics, thus the response distributions were used.

4. The location of a color sample on the computer screen has an effect on preferences.

5. Gender has an effect on color preferences.

\section{THE EXPERIMENT}

\section{Experimental Setup}

The experimental setup consisted of a computer monitor in a windowless room illuminated with cove lighting (Fig. 1). Cove lighting was preferred as it excluded the possibility of glare on the monitor and created a perfectly diffuse environment without any highlights that might have distracted the subjects. Standard Philips TL 54 fluorescent with a 6200 color temperature (CT) and a 72 color rendering index (CRI) was used in the coves for lighting the room.

The computer monitor was set to $1024 \times 768$ HiColor (16 bit), all desktop patterns were turned off, and the background color on the monitor was set to a light gray (hue $=$ 0 , saturation $=0$, luminance $=200$; red $=212$, green $=212$, blue $=212$ ). The calibration was set as: contrast $=$ 240 ; brightness $=230 ; \mathrm{B}=140$. The Photoshop monitor setup was: gamma $=2.0$; white point $=6500^{\circ} \mathrm{K}$; phosphors $=$ Trinitron; ambient light $=$ medium; gamma (calibrate) $=12$; white point: all $\mathrm{RGB}=255$; balance: all $\mathrm{RGB}=0$; black point: all $\mathrm{RGB}=0$. The display was spatially uniform and channel independent, which was tested with chromameter measurements.

TABLE II. Hues that are most preferred on specified backgrounds.

\begin{tabular}{|c|c|}
\hline Background $\mathrm{Ce}$ & Preferred Color \\
\hline Red & Blue $(35 \%)$ \\
\hline Yellow & Blue (23\%), Red (17\%) \\
\hline YellowGreen* & Blue (19\%) \\
\hline Green* & Blue $(21 \%)$ \\
\hline Cyan & Blue (44\%) \\
\hline Blue & Purple (21\%), Red (22\%), Magenta (20\%) \\
\hline Purple & Magenta (36\%) \\
\hline Mingent: & Blue $(43 \%)$ \\
\hline
\end{tabular}

* No statistically significant hues were differentiated for these backgrounds, thus inclinations from response distributions have been included. 


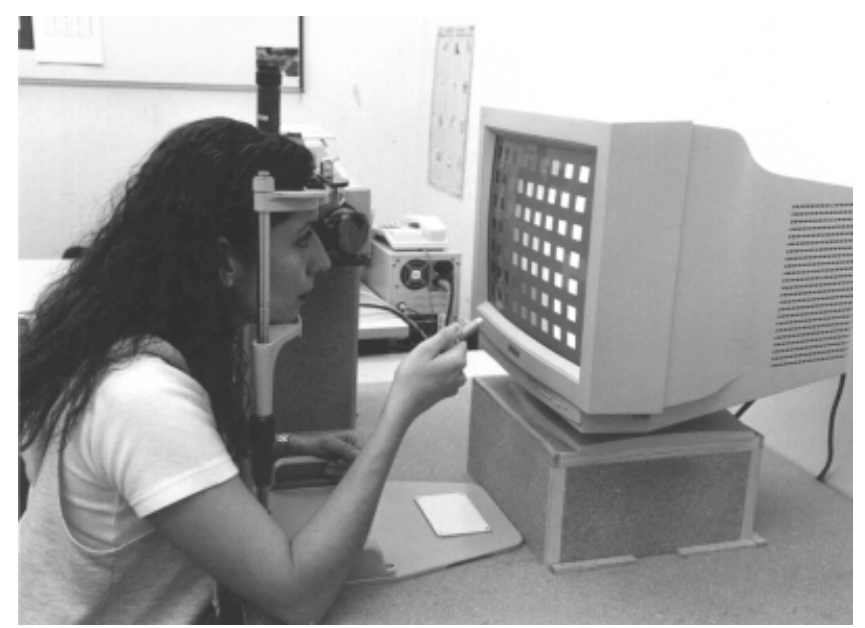

FIG. 1. Experimental setup, in which subjects view the image sets through a headrest depending on their response speed.

\section{Experimental Procedure}

One hundred twenty-three undergraduate students studying in art/design-related departments were presented image sets displayed on a computer monitor. Each image set consisted of a background color selected from HSB (hue, saturation, brightness) color space and 63 color squares of differing hues, saturations, and brightnesses. Every subject viewed and answered the experimental question for the eight background colors.

The experimenter verbally asked each participant: "Which color square would you prefer on the background color on the screen?" ${ }^{\dagger}$ No time limits were set for making the decisions. Participants were tested individually over a period of 5-15 min, depending on their response speed.

Despite the quantity of color squares from which to choose (63 color squares for each background), the participants had no difficulty in choosing one over the others. Making a single choice took about 10-15 sec for one participant, although it varied from approximately $5 \mathrm{sec}$ to $50 \mathrm{sec}$ because of variation in individual response speed. No matter how long it took to choose a single color square, none of the participants showed any difficulty or hesitation in making a selection of a single color square.

In addition to the main image set, three more image sets were shown to the participants, who answered the same question for those as well. These supplementary sets were used to investigate the effect of location of the color squares on the choices.

All the participants were students in the Faculty of Art, Design, and Architecture at Bilkent University in Ankara, Turkey. The majority were 20- to 24-year-old (78\%) female (59\%) second-year students (55\%) in the Department of Interior Architecture and Environmental Design (81\%). All

\footnotetext{
${ }^{\dagger}$ The experimental question was asked in Turkish, which may also be translated as: "Which color combination of square and background would you prefer?"
}

were from urban areas, with the majority being inhabitants of Ankara (76\%); thus, no participants were from diverse cultural backgrounds in small towns or rural areas. Participants with vision deficiencies were asked to take the test wearing the corrective equipment-contact lenses or eyeglasses-that they wore regularly. There were no participants with severe eye or vision problems, which would have required their exclusion from the test. Participants were also given Ishihara's Tests for Colour-Blindness (Ishihara). ${ }^{10}$ Anyone unable to read any of the plates shown could not participate.

\section{Image Sets}

Adobe Photoshop $4.0^{11}$ was used to create the entire image set. The screen area was adjusted to $1024 \times 768$ pixels for every image produced. All images were created in JPEG format and RGB mode. The Photoshop color picker function was used to create displayed colors. Foreground and background colors were selected from a color spectrum based on the HSB color model. HSB lets the user choose a color with a hue from $0^{\circ}$ to $360^{\circ}$, with saturation and brightness values from $0 \%$ to $100 \%$.

The main image set consisted of eight images, each with a different background color. All the background colors were fully saturated (100\% saturation) and fully bright (100\% brightness). The angle for hue was defined as an angle relative to pure red on the color circle. Hues were selected from the standard color circle beginning with red $\left(0^{\circ}\right)$, continuing at $45^{\circ}$ intervals, and ending at magenta $\left(315^{\circ}\right)$. The background colors used are (Fig. 2):

$0^{\circ}$ Red, $100 \%$ saturation, $100 \%$ brightness

$\mathbf{4 5}^{\circ}$ Yellow, $100 \%$ saturation, $100 \%$ brightness

$\mathbf{9 0}^{\circ}$ Yellow-Green, $100 \%$ saturation, $100 \%$ brightness

$\mathbf{1 3 5}^{\circ}$ Green, $100 \%$ saturation, $100 \%$ brightness

$\mathbf{1 8 0}^{\circ}$ Cyan, $100 \%$ saturation, $100 \%$ brightness

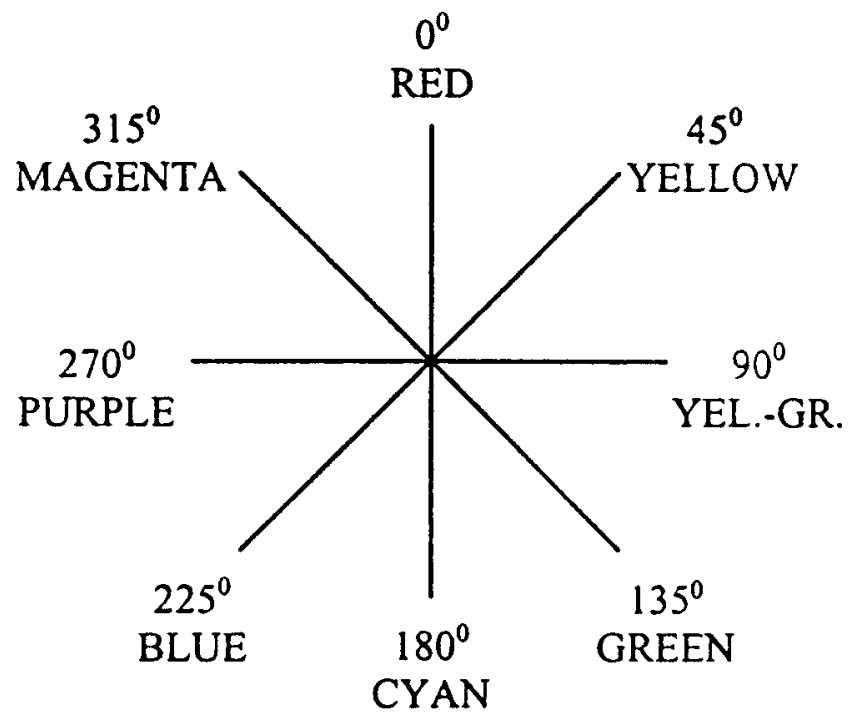

FIG. 2. Color circle used. 
$\mathbf{2 2 5}^{\circ}$ Blue, $100 \%$ saturation, $100 \%$ brightness

$\mathbf{2 7 0}^{\circ}$ Purple, $100 \%$ saturation, $100 \%$ brightness

$\mathbf{3 1 5}^{\circ}$ Magenta, $100 \%$ saturation, $100 \%$ brightness

On every background color all the remaining hues (excluding the background hue) were represented in seven rows. Each hue row was then divided into nine columns, in each of which the hue was represented with varying brightness and saturation levels. Explanations and examples of the images are included in Appendix A.

Three different supplementary image sets were prepared to test the location effect. The researcher investigated whether any particular brightness-saturation range (column) or hue range (row) was preferred independent of its place in an image. The first two image sets were scrolling sets. In one set, the rows (hues) scrolled downward and in another the columns (brightness-saturation) scrolled to the right. The last supplementary set was a rotating set, in which the whole image was rotated clockwise at $90^{\circ}$ intervals $\left(0^{\circ}\right.$, $\left.90^{\circ}, 180^{\circ}, 270^{\circ}\right)$. All the supplementary sets were applied on four background colors, which were paired as angular opposites of each other on the HSB color circle. The backgrounds used for the supplementary sets were: red $0^{\circ}$-cyan $180^{\circ}$ and yellow $45^{\circ}-$ blue $225^{\circ}$. Examples of the images for the supplementary sets are included in Appendix A.

\section{DATA ANALYSIS}

Analysis of variance (ANOVA) was used to calculate the statistics for the collected data. The randomized complete block design was used in data analysis. The data were arranged into homogeneous groups and were compared for a number of treatments. For the main image set the homogeneous groups (blocks) were different amounts of brightness-saturation levels, as presented in columns to the observer, while the treatments were differing hues, as presented in rows to the observer. A research hypothesis is considered statistically significant if the $p$ value of the test is smaller than 0.05 .

SAS (Statistical Analysis System) software ${ }^{12}$ was used in the analysis of the collected data. Each background color was analyzed for column-row effect using the ANOVA procedure, with the columns representing the brightnesssaturation and the rows representing hue. The results of the statistical analysis show that hue had an effect on preferences on specified background colors (with $p$ values between 0.0001 and 0.0209), except for yellow-green and green ( $p=0.9248$ and 0.1618 , respectively). Brightnesssaturation also had an effect on preferences on specific background colors (with $p$ values between 0.0001 and 0.0200). Duncan's multiple-range test was also applied. Pairwise comparison for each possible pair was done using this test, which provided information on the differences between the means of each individual class, brightnesssaturation and hue, for the individual background colors. The results from the ANOVA procedure and Duncan's multiple-range test are included in Appendix B, Table I.

Background effect was also investigated by making a data structure. The statistics show a brightness-saturation effect $(p=0.0001)$ and a hue effect $(p=0.0001)$ on any background color viewed. Thus, despite the changing hues of the background, certain brightness-saturation levels and certain hues were preferred over others (100\% brightnesssaturation and blue; see Appendix B, Tables II and III).

The effect of location was investigated by analyzing the responses for the viewed individual background colors. In these images the positions of the color squares were changed while the background color remained the same. Responses were analyzed statistically for each brightnesssaturation and hue range for every image shown. The results indicate a hue effect (with $p$ values between 0.0001 and 0.0014 ) and a brightness-saturation effect (with $p$ values between 0.0001 and 0.0011 ), despite the changing locations of the hues and brightness-saturation levels on the computer screen (Appendix B, Tables IV-VII).

An ANOVA analysis was done to investigate gender effect for the main image set. The results revealed no gender effect on preference choices (with $p$ values between 0.0604 and 0.2215; see Appendix B, Table VIII).

\section{DISCUSSION}

Analyzing the data required an interpretation of all the $p$ values obtained from the main and supplementary sets (Appendix B). Sometimes the data from the main image set did not significantly differentiate among any color attributes (hue, saturation, brightness), but statistically significant groupings were found for supplementary sets. As the colors shown for every image and the subject group were the same, all statistics were interpreted as a whole. The tables and figures indicating the most preferred color attributes contain statistically significant attributes obtained from Duncan's analysis. If there were no differentiated attribute from Duncan's analysis, but the attribute was still statistically significant, the attributes of the highest percentage from the data for that image were included. All the percentages given were obtained from the main image set.

The BS100 range was significantly preferred by the participants (with $p$ values between 0.0001 and 0.0200; Appendix B, Table I). Therefore, it was found that the brightest and most saturated color squares were preferred on the brightest and most saturated background colors. This finding supports the results of experiments with isolated colors. Guilford, ${ }^{5}$ Smets, ${ }^{6}$ Guilford and Smith, ${ }^{13}$ and Sivik ${ }^{14}$ all stated that isolated colors that were brighter and more saturated were more preferred. Only Eysenck ${ }^{7}$ stated a contradicting result, that brighter colors were less preferred. Studies on color combinations, especially the ones with foreground-background color relationships, suggest brightness contrast. Washburn and Grose ${ }^{15}$ and Reddy and Bennett $^{16}$ demonstrated that in the judgment of experiment participants, the more brightness contrast was increased, the more preferred was a color combination. This argument cannot be evaluated within the scope of this study because in participant preferences there was no brightness contrast between the background and foreground colors and the 


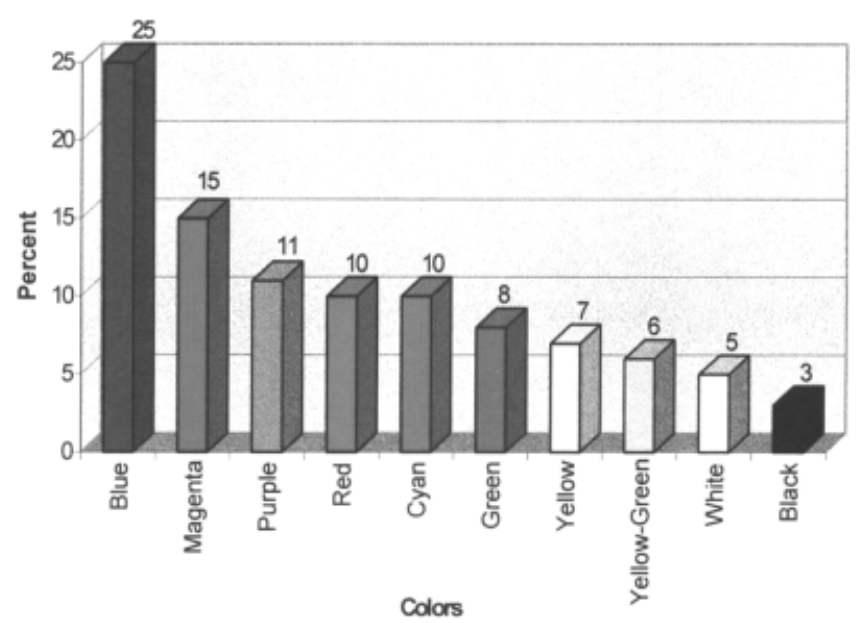

FIG. 3. Percentage scale of preference for hues on any background color.

background colors were presented as a predetermined condition. However, in much of the literature on color combinations how various attributes of background colors enhance the pleasantness of foreground colors with varying background presentations has been investigated. One exception to the preferred BS100 range in the current study was seen with the yellow background, where brightness levels of $\mathrm{B} 50$ and B75 were also preferred along with $\mathrm{BS} 100(p=$ 0.0001, Appendix B, Table VI). On the purple background saturation levels of S50 and S75 were preferred along with BS100 ( $p=0.0002$, Appendix B, Table I). Another exception was observed for the cyan background, for which saturation levels of S25 and S50 also were preferred along with BS100 ( $p=0.0001$, Appendix B, Table V). In the third supplementary image set, a saturation of S25 was preferred over the other brightness-saturation levels for the cyan background ( $p=0.0001$, Appendix B, Table VI).

Regardless of the background colors viewed, the subjects preferred blue the most [Fig. 3 ( $p=0.0001$, Appendix B, Table III)]. This corresponds with the findings of previous experimental research. Wijk et al., ${ }^{17}$ Guilford, ${ }^{5}$ Eysenck, ${ }^{7}$ Granger, ${ }^{18}$ and Guilford and Smith ${ }^{13}$ all found that blue was preferred the most when isolated colors were presented. Sivik ${ }^{14}$ stated blue was the color preferred in more instances despite its change in brightness and saturation. Saito ${ }^{19}$ stated that blue was named most when people were asked the color they liked best. Washburn and Grose ${ }^{15}$ found that blue was the most preferred color regardless of its background.

Data gathered in this inquiry demonstrated that green $(8 \%)$, yellow (7\%), yellow-green $(6 \%)$, and the noncolors white $(5 \%)$ and black (3\%) were the hues preferred the least (Fig. 3). The studies by Guilford, ${ }^{5}$ Eysenck, ${ }^{7}$ Granger, ${ }^{18}$ Guilford and Smith, ${ }^{13}$ and Washburn and Grose ${ }^{15}$ also stated yellow was the least preferred hue.

Blue was preferred on background colors of red, cyan, and magenta. Magenta was preferred the most on the purple background (Appendix B, Table I). For the yellow and blue background colors it was hard to determine if there was any agreement on preference. The results from the main image set for the yellow background showed a scattered distribution of preferences. The first supplementary set (scrolling down) statistically showed a preference for blue, while the last supplementary set (rotating) favored red as the preferred hue on a yellow background. The same participants viewed all the image sets. Thus, it can be concluded that yellow is a difficult background on which to make a hue preference, although there is a considerably significant inclination toward preferring blue and/or red. A similar difficulty in deciding on preferences was found for the blue background. No preference was statistically significant for any color set on the blue background in the main image. The results of the first supplementary image set statistically singled out purple as the most preferred hue on a blue background. The results for the last supplementary set (rotating) showed red, magenta and purple as the preferred hues on blue (Appendix B, Tables IV and VII). The yellow-green and green backgrounds had scattered response distributions. Although the participants had no statistically significant hue preference on the yellow-green and green backgrounds, there was an inclination towards blue on these backgrounds.

The findings on foreground-background color relationships presented above do not support the views of Granger, ${ }^{18}$ Chevreul, ${ }^{2}$ and Itten ${ }^{4}$ concerning hue combinations. There does not seem to have been a harmony of either analogous or contrasting colors in making preference judgments. It may be interesting to research the issue further by excluding the dominant blue from the image sets. The exclusion of blue may direct subjects to suggest different color combinations, or it may simply promote another dominant hue.

The results of the statistical analysis show that in this experiment neither the gender of the participant nor the location of color squares on the computer screen had a significant effect on color preference (Appendix B, Tables IV-VIII).

\section{Findings on the Research Hypotheses}

1. Hue has an effect on preferences on a specific background color.

2. Varying brightness-saturation levels of color samples have an effect on preferences on a specific background color.

3. Despite the changing hues of the background, some brightness-saturation levels and some hues are preferred over others.

4. Despite the changing locations of color samples on the computer screen, some brightness-saturation levels and some hues are preferred over others.

5. Gender does not have an effect on color preference.

\section{CONCLUSION}

Analyses of data demonstrated that all three attributes of color (hue, saturation, and brightness) were important in preferences. Colors having maximum brightness and maximum saturation levels were ranked higher than any other 
brightness-saturation combination. Previous experimental studies on isolated colors also have suggested an increase in rated pleasantness with an increase in brightness and saturation.

Blue was the most preferred hue regardless of background shown. This confirms the findings of previous studies (Wijk et al., ${ }^{17}$ Guilford, ${ }^{5}$ Eysenck, ${ }^{7}$ Granger, ${ }^{18}$ Guilford and Smith ${ }^{13}$ ), which found that blue appeared to be the preferred color in isolation. In the present study analyses of individual backgrounds were tabulated according to most preferred hues on specified background colors (Table II).

The results of the statistical tests showed that location and gender had no significant effect on preference in this experiment.

The findings showed that the preferred color choices, although statistically significant, encompassed a low percentage of the subjects. The choices did not encompass $45 \%$ of the respondents and were usually around $20 \%$. This may suggest a variety in preference choices.

Color has always been considered to be linked with subjectivity, especially where preferences are concerned. Culture also is always believed to have an influence, as is gender. The general preference found for blue in this study supports the findings of previous studies having subject groups of varied cultures. No matter what the background, blue was most preferred. There seems to be a global inclination toward preferring blue regardless of its presented medium. In many cases, blue was also stated as the most likeable color when people were asked their favorite color without any visual stimuli. This finding contradicts the views on color combinations of the experts, who had formulated numerous color pairs based on the premise that pleasurable combinations result from the hue component of color.

The attributes of brightness and saturation seem to play a great role in creating pleasurable color combinations in abstract representations. The results of this study demonstrated that foreground colors were preferred when their saturation and brightness levels were increased. The participants picked the maximum levels available. The literature review suggests brightness and saturation contrasts to be effective for preference in foreground-background color relationships. It may be interesting to reverse this experiment and present backgrounds of the same hue with varying brightness and saturation levels to find out on which background a specific color square is preferred more. In this way, additional knowledge about the contrast relations between background and foreground may be gained.

Next it is intended to conduct a similar experiment with CIELAB color space and compare the obtained results with those for HSB color space.

The conclusions of this study should be used with caution, taking into account the limitations and delimitations of the experimental setup, and the characteristics of the subject group involved. The results of the study may be used in computer applications such as computer graphics and Web page design and in luminous signage applications. Moreover, we believe the study has contributed to the available data on this subject by broadening the understanding of color preferences.

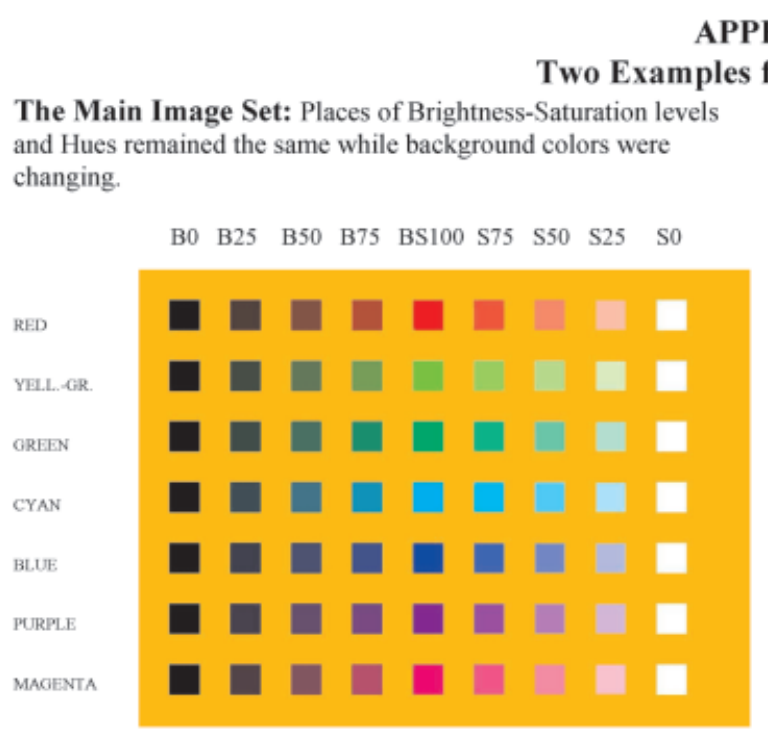

Yellow Background

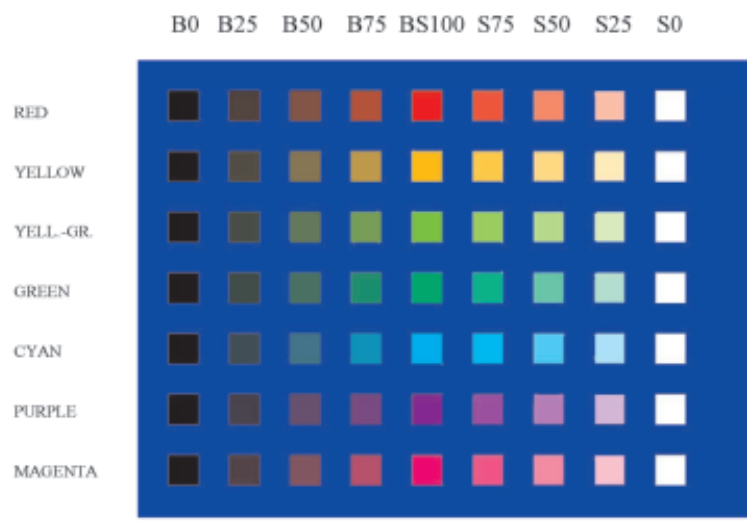

$\underline{\text { Blue Background }}$

The First Supplementary Image Set (scrolling down): Places of Hues were changing in each image, moving towards one row below. For example, yellow in the first row in the first image would move to the second row in the second image, etc. 
APPENDIX A (CONTINUED)

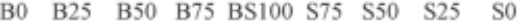

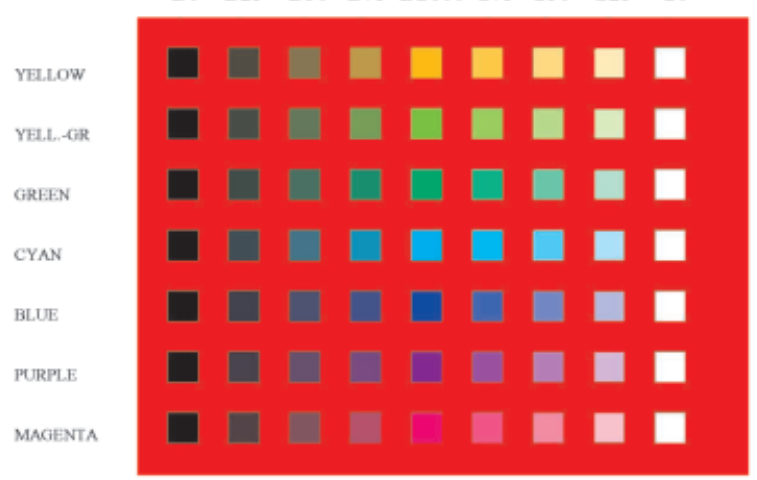

$\underline{\text { Red Background/ } 1^{\text {st }} \text { Image }}$

The Second Supplementary Image Set (Scrolling to the right): Places of Brightness-Saturation levels were changing in each image, moving towards one column to the right. B25 in the first column in the first image would move to the second column in the second image, etc.

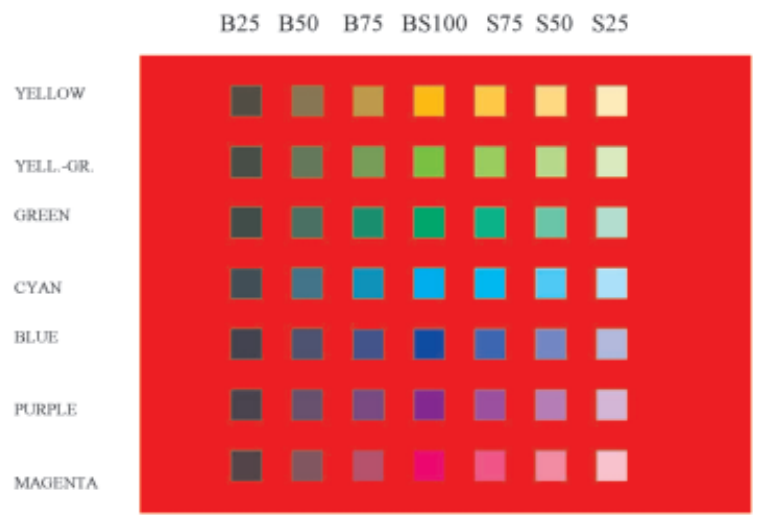

$\underline{\text { Red Background/ } 1^{\text {st }} \text { Image }}$

The Third Supplementary Image Set (Rotating): Images were rotated $90^{\circ}$ clock-wise, where places of both

Brightness-Saturation levels and Hues were changing with each image.

B25 B50 B75 BS100 S75 S50 S25

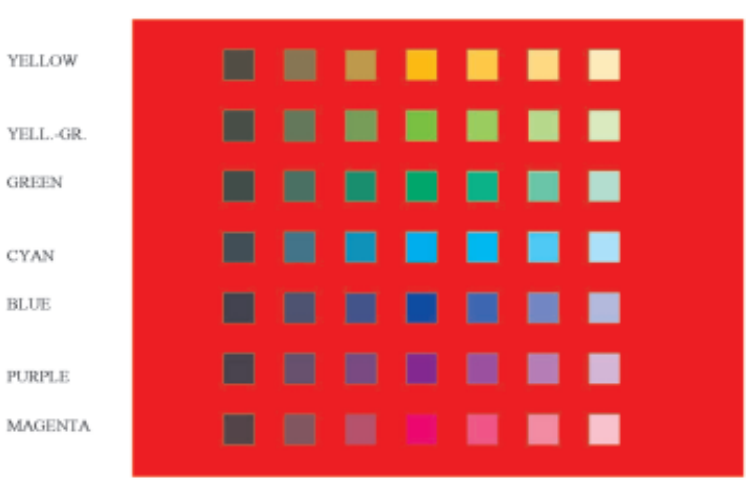

$\underline{\text { Red Background/ } 1^{\text {st }} \text { Image }}$

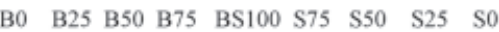

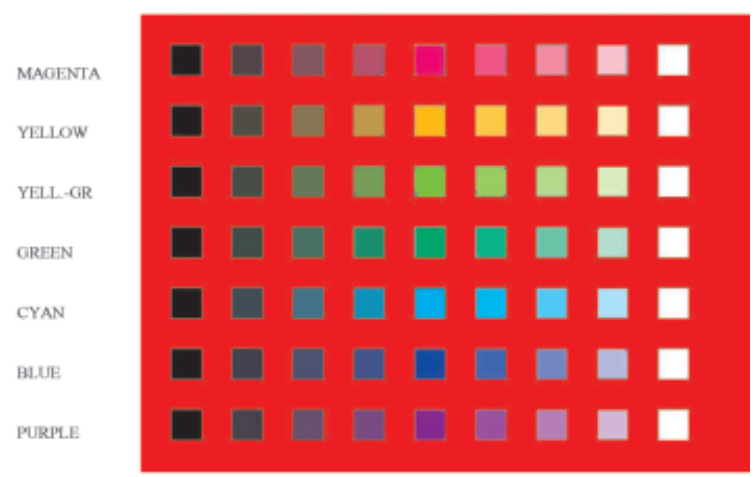

$\underline{\text { Red Background } / 2^{\text {nd }} \text { Image }}$

S25 B25 B50 B75 BS100 S75 S50

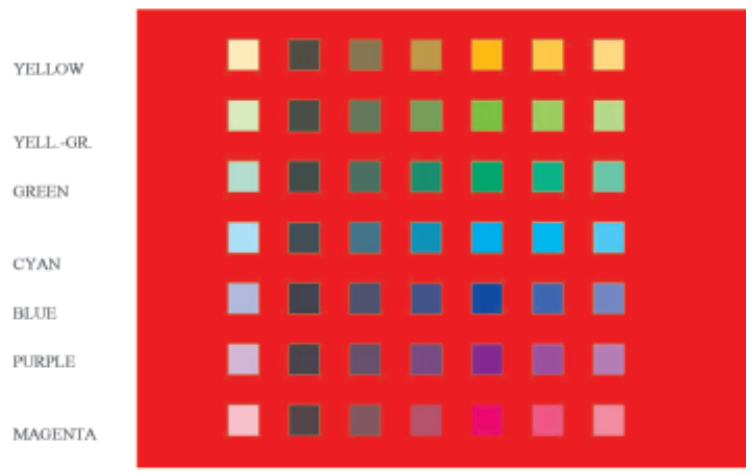

$\underline{\text { Red Background } / 2^{\text {nd }} \text { Image }}$

MAGEN PURP BL CYAN GR YELL-GR YELL.

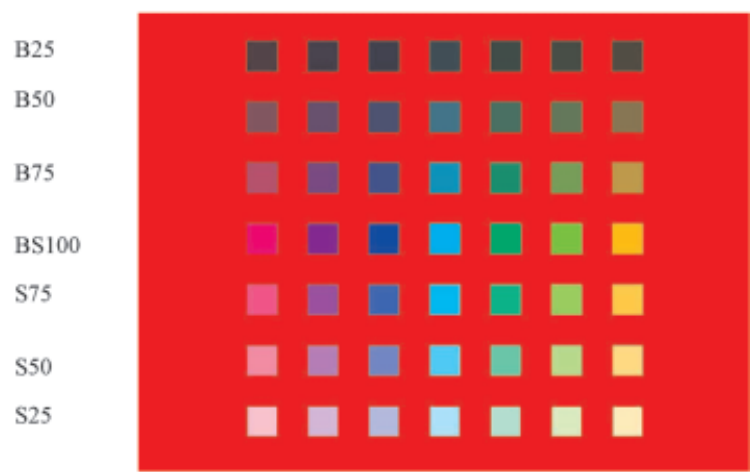

$\underline{\text { Red Background } / 2^{\text {nd }} \text { Image }}$ 
APPENDIX B

$p$-Values and Duncan Groupings

TABLE I. Brightness-saturation and hue effect.

\begin{tabular}{lcccc}
\hline Background colors & $\begin{array}{c}p \text { values for } \\
\text { brightness-saturation }\end{array}$ & $\begin{array}{c}\text { Duncan grouping for } \\
\text { brightness-saturation }\end{array}$ & $\begin{array}{c}p \text { values } \\
\text { for hue }\end{array}$ & $\begin{array}{c}\text { Duncan grouping } \\
\text { for hue }\end{array}$ \\
\hline Red & 0.0128 & Overlapping & 0.0002 & H225-Blue \\
Yellow & 0.0003 & Overlapping & 0.0209 & Overlapping \\
Yellow-green & 0.0033 & Overlapping & 0.9248 & None different \\
Green & 0.0001 & BS100 & 0.1618 & Overlapping \\
Cyan & 0.0084 & Overlapping & 0.0001 & H225-Blue \\
Blue & 0.0001 & BS100 & 0.0064 & Overlapping \\
Purple & 0.0002 & BS100, S50, S75 & 0.0004 & H315-Magenta \\
Magenta & 0.0200 & Overlapping & 0.0001 & H225-Blue \\
\hline
\end{tabular}

TABLE II. Brightness-saturation and background effect.

\begin{tabular}{lcc}
\hline & $\begin{array}{c}p \text { values for } \\
\text { brightness-saturation }\end{array}$ & $\begin{array}{c}\text { Duncan grouping for } \\
\text { brightness-saturation }\end{array}$ \\
\hline $\begin{array}{l}\text { On any background } \\
\text { color }\end{array}$ & 0.0001 & BS100 \\
\hline
\end{tabular}

TABLE III. Hue and background effect.

\begin{tabular}{lcc}
\hline & $\begin{array}{c}p \text { values } \\
\text { for hue }\end{array}$ & $\begin{array}{c}\text { Duncan grouping } \\
\text { for hue }\end{array}$ \\
\hline On any background color & 0.0001 & H225-blue \\
\hline
\end{tabular}

TABLE IV. Hue effect for the first supplementary image set (scrolling down).

\begin{tabular}{lll}
\hline Background colors & $\begin{array}{c}p \text { values } \\
\text { for hue }\end{array}$ & $\begin{array}{c}\text { Duncan grouping } \\
\text { for hue }\end{array}$ \\
\hline Red & 0.0001 & H225-blue \\
Yellow & 0.0001 & H225-blue \\
Cyan & 0.0001 & H225-blue \\
Blue & 0.0001 & H270-purple \\
\hline
\end{tabular}

TABLE VII. Hue effect for the third supplementary image set (rotating).

\begin{tabular}{lll}
\hline $\begin{array}{l}\text { Background } \\
\text { colors }\end{array}$ & $\begin{array}{c}p \text { values } \\
\text { for hue }\end{array}$ & Duncan grouping for hue \\
\hline Red & 0.0014 & H225_blue \\
Yellow & 0.0001 & H0_red \\
Cyan & 0.0001 & H225—blue \\
Blue & 0.0001 & H0_red, H315-Magenta, \\
& & H270-Purple \\
\hline
\end{tabular}

TABLE V. Brightness-saturation effect for the second supplementary image set (scrolling to the right).

\begin{tabular}{lcl}
\hline $\begin{array}{c}\text { Background } \\
\text { colors }\end{array}$ & $\begin{array}{c}p \text { values for } \\
\text { brightness-saturation }\end{array}$ & $\begin{array}{c}\text { Duncan grouping for } \\
\text { brightness-saturation }\end{array}$ \\
\hline Red & 0.0001 & BS100 \\
Yellow & 0.0011 & Overlapping \\
Cyan & 0.0001 & S50, S25, BS100 \\
Blue & 0.0001 & BS100 \\
\hline
\end{tabular}

TABLE VI. Brightness-saturation effect for the third supplementary image set (rotating).

\begin{tabular}{lll}
\hline $\begin{array}{c}\text { Background } \\
\text { colors }\end{array}$ & $\begin{array}{c}p \text { values for } \\
\text { brightness-saturation }\end{array}$ & $\begin{array}{c}\text { Duncan grouping for } \\
\text { brightness-saturation }\end{array}$ \\
\hline Red & 0.0005 & BS100 \\
Yellow & 0.0001 & BS100, B50, B75 \\
Cyan & 0.0001 & S25 \\
Blue & 0.0001 & BS100 \\
\hline
\end{tabular}

TABLE VIII. Gender effect on the main image set.

\begin{tabular}{lcc}
\hline $\begin{array}{c}\text { Background } \\
\text { colors }\end{array}$ & $\begin{array}{c}p \text { values } \\
\text { for gender }\end{array}$ & $\begin{array}{c}\text { Duncan grouping } \\
\text { for gender }\end{array}$ \\
\hline Red & 0.0667 & None different \\
Yellow & 0.0604 & None different \\
Yellow-green & 0.1465 & None different \\
Green & 0.0635 & None different \\
Cyan & 0.1106 & None different \\
Blue & 0.0686 & None different \\
Purple & 0.2215 & None different \\
Magenta & 0.1482 & \\
\hline
\end{tabular}




\section{LIST OF ABBREVIATIONS}

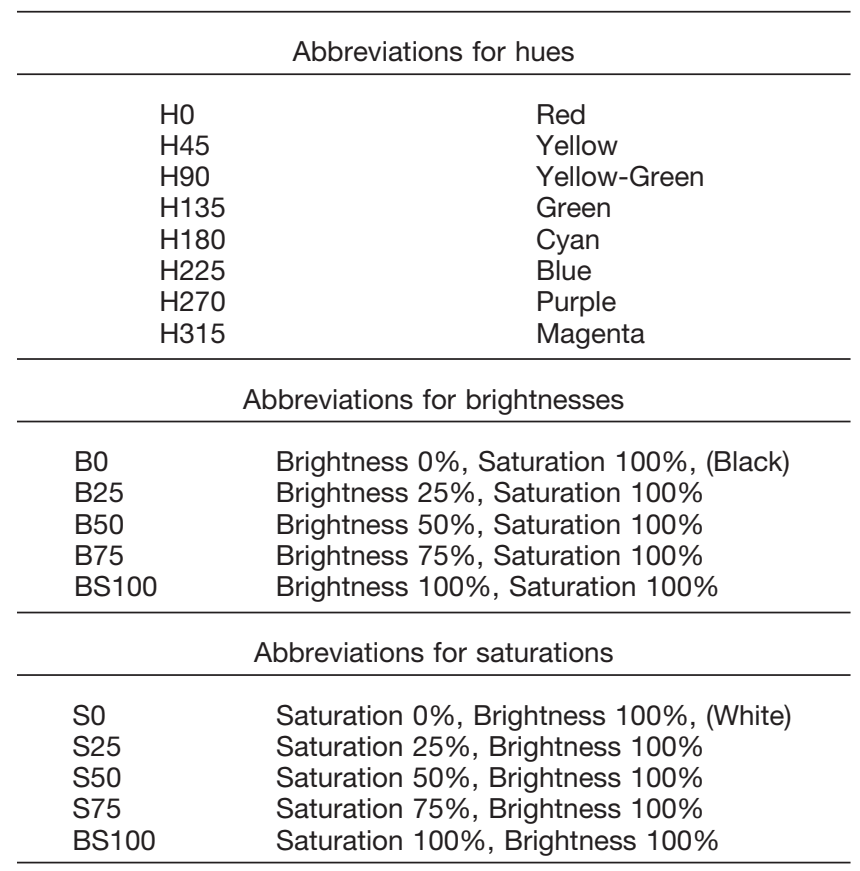

1. Birren F. Color and human response. New York: Van Nostrand Reinhold; 1978.

2. Chevreul ME. The principles of harmony and contrast of colors and their application to the arts. Rev. ed. West Chester (PA): Schiffer; 1987.

3. Munsell AH. A grammar of color. Birren F, editor. New York: Van Nostrand Reinhold; 1969.

4. Itten J. The elements of color. Van Hagen E, translator; Birren F, editor. New York: Van Nostrand Reinhold; 1970.
5. Guilford JP. The affective value of color as a function of hue, tint and chroma. J Exp Psychol 1934;17:342-370.

6. Smets G. A tool for measuring relative effects of hue, brightness and saturation in color pleasantness. Perceptual and Motor Skills 1982; 1159-1164.

7. Eysenck HJ. A critical and experimental study of color preferences. Am J Psychol 1941;54:385-391.

8. Helson H, Lansford T. The role of spectral energy of source and background color in the pleasantness of object colors. Appl Opt 1970;9:1513-1562.

9. Camgöz N. Effects of hue, saturation, and brightness on attention and preference [dissertation]. Ankara (Turkey): Bilkent University; 2000.

10. Ishihara S. Ishihara's tests for colour-blindness. Concise ed. Tokyo: Kanehara Shuppan; 1975.

11. Adobe Photoshop [computer program]. Version 4.0. San Jose (CA): Adobe Systems; 1996.

12. SAS [computer program]. Version 6.12. SAS/STAT User's guide. Release 6.03 ed. Cary (NC): SAS Institute; 1999.

13. Guilford JP, Smith PC. A system of color-preferences. Am J Psychol 1959;72:487-502.

14. Sivik L. Studies of color meaning. Man-Environment Systems 1975; 5:155-160.

15. Washburn MF, Grose SL. Voluntary control of likes and dislikes: the effects of an attempt voluntarily to change the affective value of colors. Am J Psychol 1921;32:284-289.

16. Reddy TV, Bennett CA. Cultural differences in color preferences. Proceedings of the Human Factors Society, 29th annual meeting; 1985. p 590-593.

17. Wijk H, Berg S, Sivik L, Steen B. Color discrimination, color naming and color preferences in 80-year olds. Aging-Clinical and Experimental Research 1999;11(3):176-185.

18. Granger GW. An experimental study of color preferences. J Gen Psychol 1955;52:3-20.

19. Saito M. Blue and 7 phenomena among Japanese students. Perceptual and Motor Skills 1999;89(2):532-536. 